\title{
Wireless Power and Signal Transmission Based on Time-sharing Multiplexing for WPT System
}

\author{
Xiaorui $\mathrm{Wu}^{1}$, Wenlan Gong ${ }^{1}$, Jing Xiao ${ }^{1,}$, , Shaonan $\mathrm{Chen}^{1}$, Ning $\mathrm{Wu}^{2}$ \\ ${ }^{1}$ Southern Power Grid Corporation Wireless Power Transmission Joint Laboratory Guangxi Power Grid Co., Ltd, Nanning, 530023, \\ China \\ ${ }^{2}$ Electric Power Research Institute Guangxi Power Grid Co., Ltd, Nanning, 530023, China
}

\begin{abstract}
In wireless power transfer (WPT) systems, wireless transmission of signals is particularly important for some practical applications. An effective approach of signal and energy synchronous transmission based on time-sharing multiplexing of the same coupled coil is proposed in this paper. Using the same coupled coil, the signal transmission is carried on when interruption of power transmission happens. To realize the proposed method, the relationship between power transmission time and signal transmission time is provided to obtain the optimal transmission process of the power and signal. Besides, the power transmission efficiency (PTE) and the signal transmission rate (STR) are analyzed. Finally, the effectiveness of the proposed method is verified by the experiment.
\end{abstract}

\section{INTRODUCTION}

Wireless power transfer (WPT) technology allows for electrical power transmission between the power supply and the electrical equipment without physical contact 1 . Due to its residue-free operation and tolerance to harsh environment, WPT technology has received widespread attention and rapid development. In the biomedical fields, the application of WPT technology has been a great progress for human health care 2-3. In the field of consumer electronics such as household appliances and removable electronic products 45 , wireless charger is becoming popular and several standards have been set up. And in the high-power applications such as electrical vehicles (EVs), high-speed trains and oil drilling 611, the WPT technology enhances the devices' safety and decreases the connectivity complexity.

However, for many applications, the communication between the power supply side and the pickup side is always required and plays an essential role in a welldesigned ICPT system. For instance, the function such as output voltage feedback control, status monitoring and multi-controller synchronization is always required in WPT systems 12-14, and the implementation will be easier if the communication between both sides is provided. Radio frequency (RF) technology is a common way employed in many WPT systems for wireless communication 15 , but it also leads to higher costs and lower reliability with increasing the power rating of WPT systems. The dual-band system which uses multiple inductive links is also a solution in many WPT systems [16]. But multiple links will cause extra magnetic interferences and leads to larger device size.
Several methods have been proposed to transfer the power and signal via the same inductive link where the power transmission coupling coils are used as the shared channel for power and signal transmission. For example, in some applications, the signal is directly modulated by changing the output voltage amplitude or the working period of the inverter circuit, and the load-shift keying (LSK) technology is adopted to achieve the backward signal transmission 17-19. However, the method that changes the output voltage amplitude or the working period of the inverter circuit has two major drawbacks: the first one is that the power transmission performance will be affected when the working condition of the inverter circuit is changed, which causes the frequency of the inverter circuit and the power transmission capacity both to be changed; the second one is that the signal is modulated by changing the power transmission state so that the signal transmission will be disturbed when the system is disturbed or the load is changed. Besides, literature 20 realizes signal transmission by increasing the circuits of injection and receiving signal carrier, and adds the wave trapper to avoid the interference while the wave trapper will affect the system performance and increase the cost. In some low power cases, the signal is directly modulated as the highfrequency signal carrier, and the carrier is transferred in the power transmission link as with the power carrier 21 . However, the carrier will be weakened by the filtering of the resonance compensation circuit of power transmission, and the carrier will bring harmonic interference in the power transmission link.

This paper focuses on how to apply the power and signal transmission technology in oil drilling application. An effective approach based on the time-sharing

" Corresponding author: xueshudaniu@163.com 
multiplexing technology to transfer the power and signal via the same inductive link is proposed. There will not have the interference between power transmission and signal transmission because of the different work period. The distribution principle of the power transmission time and the signal transmission time is discussed to achieve the stable power transmission as well as reliable signal transmission. The system topology and the signal modulation and demodulation method are provided. Moreover, the PTE and the STR are discussed. Finally, an experimental system was designed to test the correctness of the proposed approach.

\section{SYSTEM FUNDAMENTALS}

The proposed system can be divided into two modes: the power transmission mode and the signal transmission mode. When the system works at power transmission mode, the transmission process is same as the normal WPT system. When the system works on signal transmission mode, the transmission process is similar to the method that uses the signal carrier to achieve the signal transmission.

\subsection{System Topology}

The SS, SP, PS, PP structures are basic topology for most WPT systems according to the different LC branches (series and parallel) in the primary side and the pick-up side. However, for some applications such as the oil drilling, the air gap between the primary side and the pickup side is only $2 \mathrm{~mm}$ or less, and the coupling coefficient is always higher than 0.8 . Thus, the topology without the compensation capacitor in the pick-up side is used 20. In this paper, the SN (primary series, secondary no compensation capacitor) is employed to analyze the proposed system, and the topology is shown in Fig. 1. $E_{D C}$ is the power supply, $u_{p}$ is the inverter output voltage by neglecting the higher-order harmonics. $L_{p}$ and $R_{p}$ are the inductor and the resistor of the primary inductive coil, and $C_{p}$ is the series compensation capacitor. $K_{1}$ and $K_{2}$ are the function switches. $L_{s}$ and $R_{s}$ are the inductor and the resistor of the pick-up inductive coil, $C_{f}$ is the filter capacitor, and $R_{L}$ is the load. $R_{1}$ and $R_{2}$ are the sampled resistors to extract the signal carrier from the coupling coil.

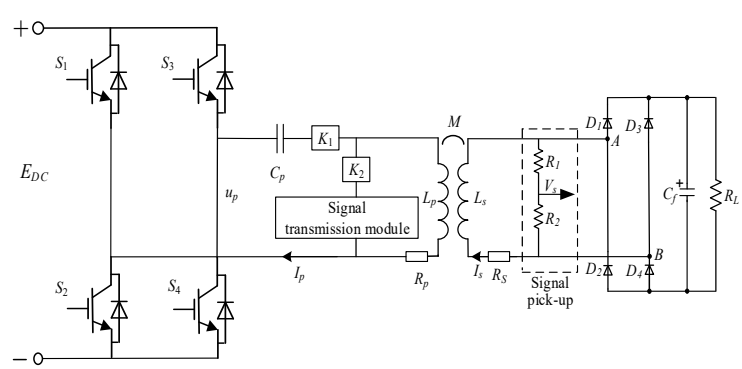

Fig. 1. The proposed system of power and signal transmission

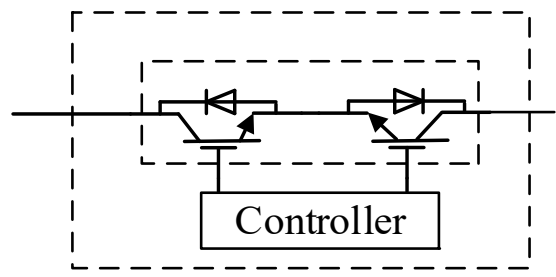

Fig. 2. The structure of function switch

In order to switch the operation mode, the function switches $K_{1}$ and $K_{2}$ are connected to the power transmission circuit and the signal transmission circuit respectively and the structure of the function switch is shown in Fig.2. The switches are controlled by a MCU. When the system works on power transmission mode, the switch $K_{l}$ in the power transmission circuit is turned on while the switch $K_{2}$ in the signal transmission circuit is turned off. When the system works at signal transmission mode, the switch $K_{l}$ will be turned off while the switch $K_{2}$ will be turned on.

\subsection{Characteristic of Inductor Current}

As shown in Fig.1, the induced voltage of the pick-up coil is supplied to the load through a full bridge rectifier and filter capacitor $C_{f}$. Loading effect of the load $R_{L}$ and the rectifier on the secondary circuit can be replaced by an equivalent load $R_{e q} 6$, which is approximately equivalent as:

$$
R_{e q}=\frac{8}{\pi^{2}} R_{L}
$$

According to a mutual inductance coupled transformer model, the induced voltage in the secondary side is related to the primary current ip. $R_{1}$ and $R_{2}$ are large resistors, so the data pick-up branch can be regarded as an open circuit for power transmission. The equivalent coupled circuit is shown in Fig. 3.

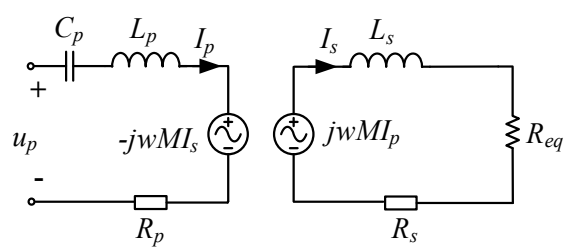

Fig. 3. The equivalent coupled circuit

According to ohm's law, the secondary current is:

$$
i_{s}=\frac{j w M I_{p}}{j w L_{s}+R_{s}+R_{e q}}=\frac{M}{L_{s}+\frac{R_{s}+R_{e q}}{j w}} I_{p}
$$

Where $w$ represents the operating frequency.

As the WPT system often operates at a highfrequency state $22,\left(R_{s}+R_{e q}\right) / j w$ as shown in (2) can be ignored in a certain power range. Then the inductor current in the secondary side can be expressed as: 


$$
I_{s}=\frac{M}{L_{s}} I_{p}
$$

Based on (3), there is no phase difference between $I_{S}$ and $I_{p}$, as shown in Fig. 4. Therefore at the time when the primary inductor current is zero, there is no energy in the secondary inductor.

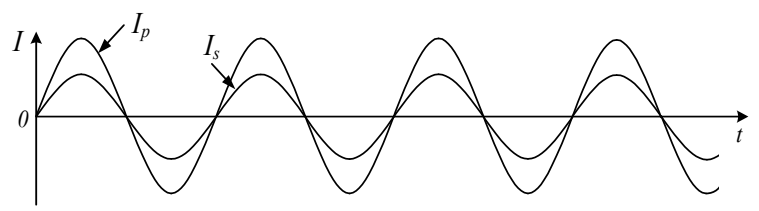

Fig. 4. The phase difference between $I_{s}$ and $I_{p}$

\subsection{Energy Characteristic of the Coupled Coils}

There is energy exchange between the inductor and the capacitor in the resonant network. When the inductor energy reaches its maximum, capacitor energy becomes zero, and vice versa. The stored energy in the inductor can be characterized by the inductor current, as shown below:

$$
W_{L}=\frac{1}{2} L i^{2}
$$

Hence, at the current zero-crossing point, the stored energy in the inductor is zero. As discussed in Section 2 .B, there is no phase difference between $i_{s}$ and $i_{p}$. Then at the primary current zero-crossing point, no energy is stored in the coupled coils.

When the inductor current is zero, the inductor can be open. At this time, energy is mainly stored in the resonant capacitor, and this state can be maintained. Furthermore, the working mode transition will occur at the time which can reduce the interference from power transmission mode to signal transmission mode.

\subsection{Signal Modulation}

At modulation cell, the on-off keying (OOK) signal modulation is used to modulate the signal. When the baseband signal is ' 1 ', the output is a continuous carrier; when the baseband signal is ' 0 ', the carrier output is closed, as shown in Fig. 5. Signal waveform formula is as follows:

$$
\begin{cases}P_{o}=A \sin \omega t & \text { singanl'1' } \\ P_{o}=0 & \text { singanl'0' }\end{cases}
$$

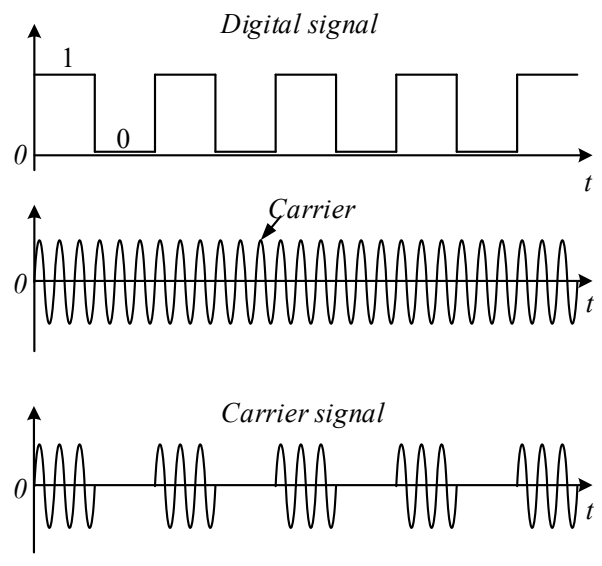

Fig. 5. Modulated waveform

\subsection{Signal Coupling}

When the switch $K_{1}$ is on and $K_{2}$ is off, the coupled coils are employed to transfer power. At the zero-crossing of the resonant current, $K_{1}$ is turned off and $K_{2}$ is turned on, then the coupled coils are used to transfer signal. After a period of time, $K_{1}$ is turned on and $K_{2}$ is turned off, then the system repeats the previous cycle. In the long term, signal and energy synchronous transmission are achieved by using a pair of coupled coils. Capacitor $C_{f}$ is used to maintain the output voltage basically stable when the signal transmission.

During signal transmission, the signal coupled voltage is lower than the output voltage of the system. Moreover, due to the full-bridge rectifier's irreversible characteristic, so the signal transmission is not affected by the output voltage in the secondary side.

\subsection{Signal Demodulation}

The sampled resistors to extract the signal carrier from the coupling coil can be analyzed as a dummy load for the whole system, so that the extracted voltage in the sampled resistors is composed of the signal carrier voltage and the power carrier voltage. Because the amplitude of the signal carrier is lower than the power carrier, so the signal carrier can be extracted by a window comparator. After the window comparator, the extracted voltage in the sampled resistors is changed into zero at the power transmission period and a series of square wave whose frequency is same as the signal carrier at the signal transmission period. Finally, the output signal of the window comparator is demodulated to the digital signal by a monostable trigger, and the block diagram of signal demodulation is shown in Fig. 6 . 


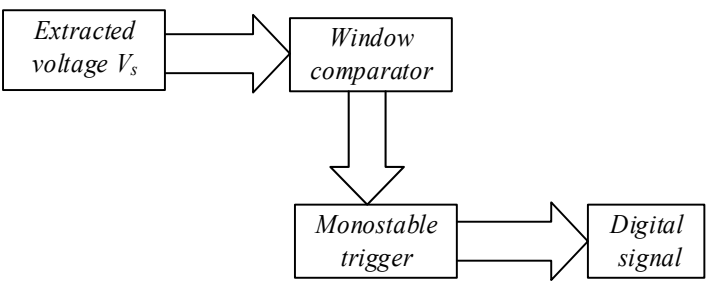

Fig. 6. The process of signal demodulation

\section{ANALYSIS OF OPERATION CYCLE}

The relationship between the power transmission period $t_{p}$ and the signal transmission period $t_{s}$ is shown in Fig.7, $t_{d}$ is the time for a data bit. In order to ensure the stable power transmission as well as the reliable signal transmission, it is necessary to analyze the power transmission process and obtain the time allocation.

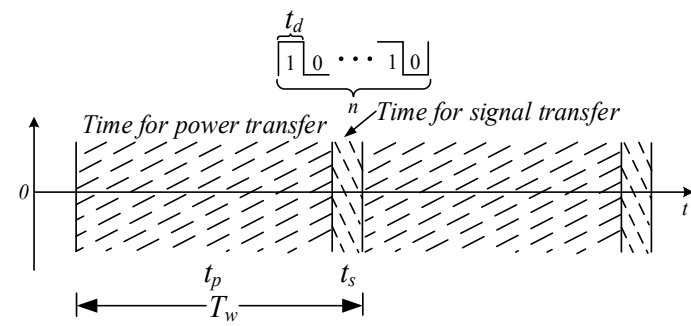

Fig.7. The operation cycle of the proposed system

\subsection{Power Transmission Efficiency}

During the power transmission, the topology of the proposed system is lack of the secondary compensation capacitor compared with the normal WPT system. The simplified model of the system is shown in Fig. 8.

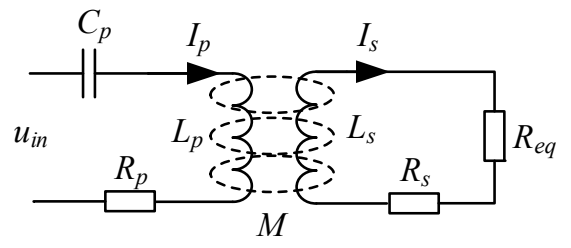

Fig. 8. The simplified model of SN network

Assuming the power inverter losses is negligible, the efficiency of system is given by 23

$$
\eta=\frac{I_{s}^{2} R_{e q}}{I_{s}^{2}\left(R_{e q}+R_{s}\right)+I_{p}^{2} R_{p}}
$$

The current $I_{p}$ and $I_{s}$ can be obtained by the following formula

$$
\left\{\begin{array}{l}
\left(R_{p}+j X_{p}\right) I_{p}-j \omega M I_{s}=U_{i n} \\
j \omega M I_{p}-\left(R_{s}+R_{e q}+j X_{s}\right) I_{s}=0
\end{array}\right.
$$

Where $\omega=2 \pi f \quad, \quad X_{p}=\omega L_{p}-1 /\left(\omega C_{p}\right)$, $X_{s}=\left\{\begin{array}{ll}\omega L_{s}-1 /\left(\omega C_{s}\right) & S S \\ \omega L_{s} & S N\end{array}\right.$.
In order to analyze the relationship of efficiency between SN topology and SS topology, the system parameters is shown in Table I.

Table 1. The parameters of system

\begin{tabular}{cc}
\hline Parameters & Values \\
\hline DC input supply $E_{d c}$ & $48 \mathrm{~V}$ \\
Primary resonant inductance $L_{p}$ & $457 \mu \mathrm{H}$ \\
Secondary resonant capacitance Ls & $456 \mu \mathrm{H}$ \\
Primary inherent resistance $R_{p}$ & $0.2 \Omega$ \\
Secondary inherent resistance $R_{s}$ & $0.2 \Omega$ \\
Mutual inductance $M$ & $422 \mu \mathrm{H}$ \\
Primary resonant inductance $C_{p}$ & $0.3 \mu \mathrm{F}$ \\
Operating frequency $f$ & $39 \mathrm{kHz}$ \\
Load resistance $R_{L}$ & $8.9 \Omega$
\end{tabular}

According to the parameters, the PTE can be obtained with respect of the normalized frequency $\left(f / f_{0}\right)$ as shown in Fig. 9.

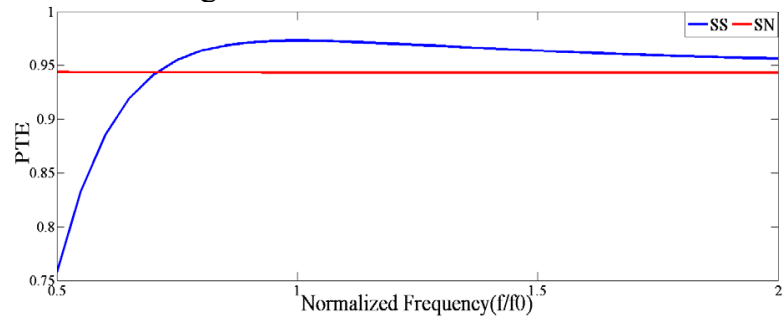

Fig. 9. PTE with respect to normalized frequency

As can be seen in Fig. 9, the maximum PTE occurs at the resonance frequency $\left(f_{0}\right)$ for SS topology. Meanwhile, the PTE remains constant almost for SN topology because of the strong coupling. So the PTE of SN decreases slightly compared with the SS topology which works at the resonance frequency.

\subsection{Signal Transmission Rate}

When the system works at signal transmission mode, the function switch $K_{1}$ is off and $K_{2}$ is on. So the inverter circuit and the compensation capacitor can be cut off from the power transmission circuit, and the signal transmission circuit is shown as Fig. 10.

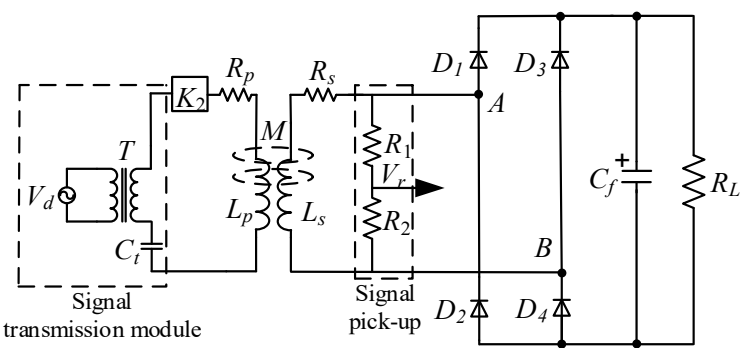

Fig. 10. The Signal transmission circuit

As shown in Fig. 10, the signal is transmitted from $V_{d}$ at the primary side and received in $V_{r}$ at the other side. $V_{d}$ and $C_{t}$ are the modulated signal and the series compensation capacitor for signal transmission, and $T$ is 
the signal coupling transformer for the signal carrier injection.

Because of the unidirectional conduction property of the rectifier diode, the rectifier circuit in the pick-up side can be analyzed as an open circuit as long as the voltage amplitude of the signal carrier in the pick-up side is lower than the output voltage across the load. So there is no need to add the switch in the pick-up side to control the working mode.

When the signal carrier frequency is high enough, signal transmission baud rate is relevant to $t_{d}, t_{s}$ and $t_{p}$, as shown in Fig. 7. Obviously, data transmission time cannot exceed $t_{s}$, which means $t_{d} \times n \leq t_{s}$. Thus, the overall STR of the system can be calculated as

$$
\text { bps }=n /\left(t_{s}+t_{p}\right)
$$

\subsection{Time Allocation}

When the system works at signal transmission mode, the output voltage can only depend on the filter capacitor $C_{f}$ to keep relatively stable. So the time allocation of $t_{p}$ and $t_{s}$ is important to ensure the realization of the function. The single-phase uncontrollable bridge rectifier circuit is shown in Fig. 11, and the output voltage $u_{o}$ waveform (red line) can be seen in Fig. 12.

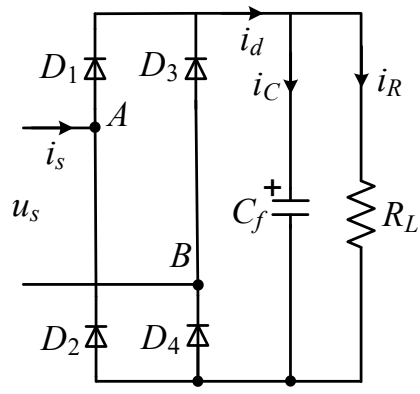

Fig. 11. The bridge rectifier circuit

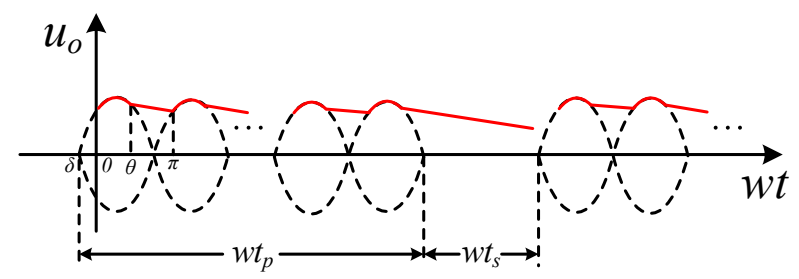

Fig. 12. The output voltage waveform

Assuming the difference between the conduction moment of $D_{1}$ and $D_{4}$ and the zero crossing point (ZCP) of $u_{s}$ is $\delta$. Only considering the fundamental harmonic of $u_{s} 22, u_{s}$ can be written in the form of

$$
u_{s}=\sqrt{2} U_{s} \sin (\omega t+\delta)
$$

During the conduction of $D_{1}$ and $D_{4}$,

$$
\left\{\begin{array}{l}
u_{o}(0)=\sqrt{2} U_{s} \sin \delta \\
u_{o}(0)+\frac{1}{C_{f}} \int_{0}^{t} i_{C} d t=u_{s}
\end{array}\right.
$$

Where $u_{o}(0)$ is the voltage value of conduction moment.

By solving (9) and (10), $i_{c}$ can be determined as

$$
i_{C}=\sqrt{2} \omega C_{f} U_{s} \cos (\omega t+\delta)
$$

And the load current $i_{R}$

So,

$$
i_{R}=\frac{u_{s}}{R_{L}}=\frac{\sqrt{2} U_{s} \sin (\omega t+\delta)}{R_{L}}
$$

$i_{d}=i_{C}+i_{R}=\sqrt{2} \omega C_{f} U_{s} \cos (\omega t+\delta)+\frac{\sqrt{2} U_{s} \sin (\omega t+\delta)}{R_{L}}$

Assuming the conduction angle of $D_{1}$ and $D_{4}$ is $\theta$, so $D_{1}$ and $D_{4}$ will be turned off when $w t=\theta$. Bringing $i_{d}(\theta)=0$ into (13), we can obtain

$$
\tan (\theta+\delta)=-\omega R_{L} C_{f}
$$

Moreover, the output voltage $u_{o}$ when $w t=\pi$ is equal to the voltage $u_{o}$ when $w t=\theta$.

$$
\sqrt{2} U_{s} \sin (\omega t+\delta) e^{-\frac{\pi-\theta}{\omega R_{L} C_{f}}}=\sqrt{2} U_{s} \sin \delta
$$

So $\theta$ and $\delta$ can be obtained by solving (14) and (15).

According to the circuit theory, the Root-MeanSquare (RMS) value of $u_{o}$ can be expressed as

$$
\begin{aligned}
& U_{o}=\sqrt{\frac{1}{T_{w}} \int_{0}^{T_{w}} u_{o}^{2}(t) d t} \\
& \text { If } \quad t_{p}=\frac{n}{2} T=\frac{n \pi}{\omega}(n=1,2,3 \ldots) \\
& \begin{cases}\sqrt{2} U_{s} \sin (\theta+\delta) e^{-\frac{\pi-\theta-\delta}{\omega R_{L} C_{f}}-\frac{t}{R_{L} C_{f}}} & -\frac{\delta}{\omega} \leq t<0 \\
\sqrt{2} U_{s} \sin (\theta+\delta) & \frac{(n-1) \pi}{\omega} \leq t<\frac{(n-1) \pi+\theta}{\omega}\end{cases}
\end{aligned}
$$

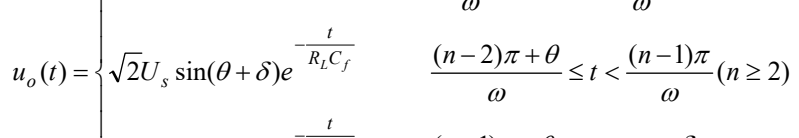

$$
\begin{aligned}
& \sqrt{2} U_{s} \sin (\theta+\delta) e^{-\frac{t}{R_{L} C_{f}}} \quad \frac{(n-1) \pi+\theta}{\omega} \leq t<\frac{n \pi-\delta}{\omega} \\
& \sqrt{2} U_{s} \sin (\theta+\delta) e^{-\frac{\pi-\theta-\delta}{\omega R_{L} C_{f}}-\frac{t}{R_{L} C_{f}}} \frac{n \pi-\delta}{\omega} \leq t<\frac{(n+m) \pi-\delta}{\omega}
\end{aligned}
$$

By using (16) and (17), we can get the expression of the RMS of $u_{o}(t)$. If $t_{p}: t_{s}=n: m=k, U_{o}$ can also be described as

$$
U_{o}=f\left(k, C_{f}\right)
$$

According to the system parameters and the above formulas, the curve of the output voltage varying with $k$ and $C_{f}$ can be obtained. In order to realize the normal work, the output voltage is $38 \mathrm{~V}$ in oil drilling application.

As can be seen in Fig. 13, under the condition of $m=4$, the $U_{o}$ is almost $38 \mathrm{~V}$ when $k=5.5$ and $C_{f}=450 \mu \mathrm{F}$, and $U_{o}$ increases with increasing $k$ and $C_{f}$. Similarly, the $U_{o}$ is $38 \mathrm{~V}$ when $k=7$ and $C_{f}=500 \mu \mathrm{F}$ in the Fig. 14 . The rest of parameter combination is no longer narration in here. 


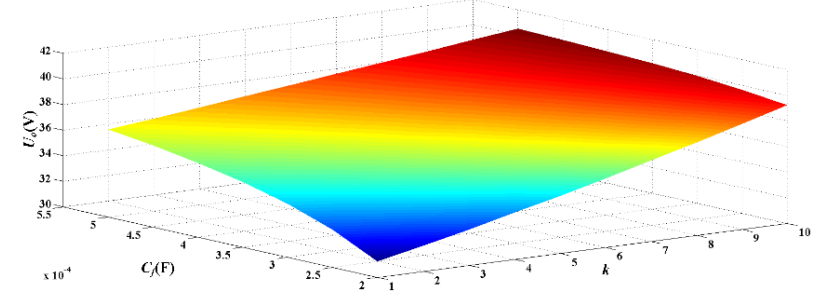

Fig. 13. The output voltage varying with $\mathrm{k}$ and $\mathrm{Cf}$ when $\mathrm{m}=4$

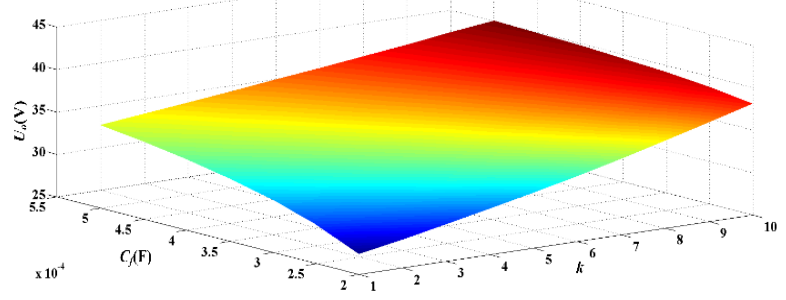

Fig. 14. The output voltage varying with $\mathrm{k}$ and $\mathrm{Cf}$ when $\mathrm{m}=6$

As can be seen from the above results, the values of $k$ and $C_{f}$ are different when signal transmission time is different. So the reasonable values of $k$ and $C_{f}$ can not only ensure the stability of power transmission, but also realize the signal transmission.

\section{EXPERIMENT VERIFICATION}

In order to verify the proposed approach, an experimental setup based on the proposed method has been built, as shown in Fig. 15. The parameters of the designed system are listed in Table I. Besides, we select $m=4, \quad k=5.5$ and $C_{f}=470 \mu \mathrm{F}$ as the experimental parameters. The experimental value of $C_{f}$ is slightly higher than the theoretical value because of the specifications.

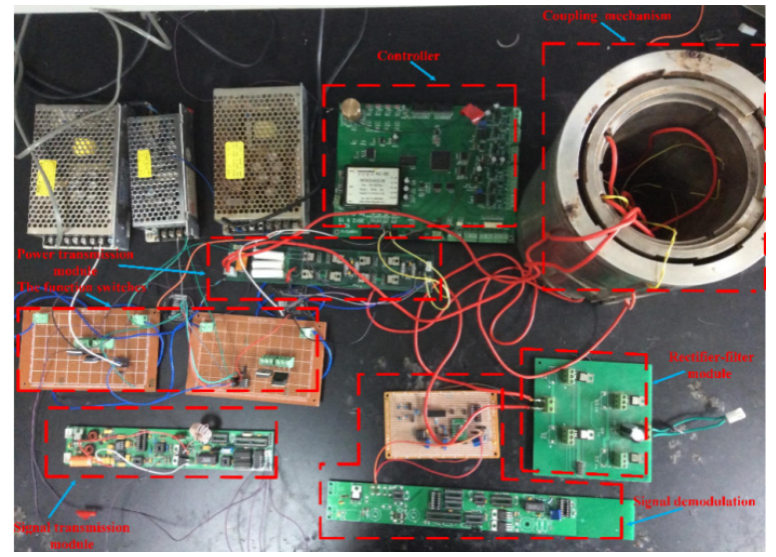

Fig. 15. The experimental equipment of the proposed system

The working mode transition occurs when the resonant current is close to zero, as shown in Fig. 16. The operating frequency is $39 \mathrm{kHz}$. The switches $K_{1}$ and $K_{2}$ work in a complementary mode. It is provided in Fig. 17 that the high-frequency carrier $(1.2 \mathrm{MHz})$ is modulated When $K_{2}$ is on. Then, the modulated waveform is coupled to the coils for signal transmission.

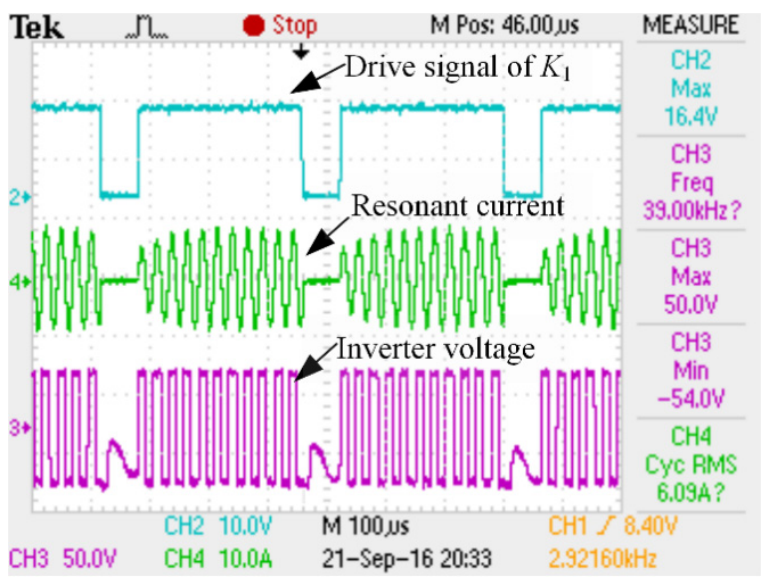

Fig.16. The inverter voltage, resonant current and drive signal of $K_{l}$

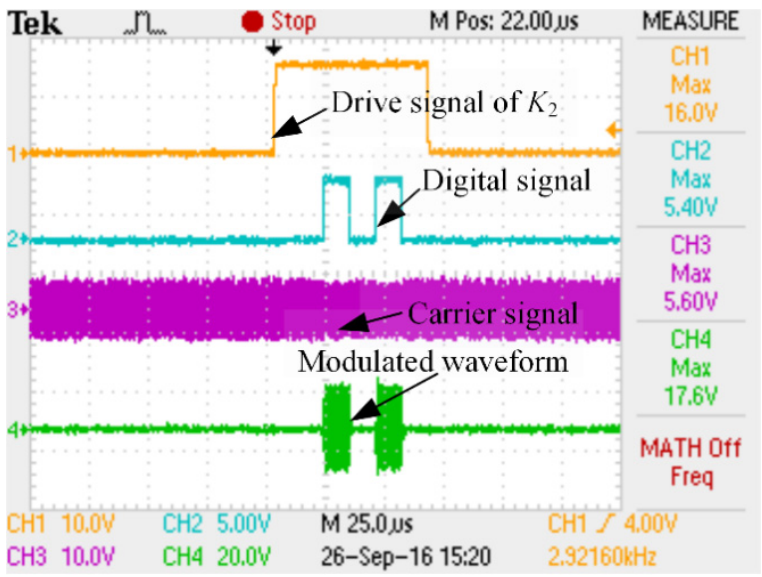

Fig.17. The signal modulated waveform

The modulated signal carrier will be transferred to the pick-up side in the signal transmission time. Due to there is no remaining power in the coupling coil, the extracted voltage in the sampled resistor only has the signal carrier. And the results are shown in Fig. 18.

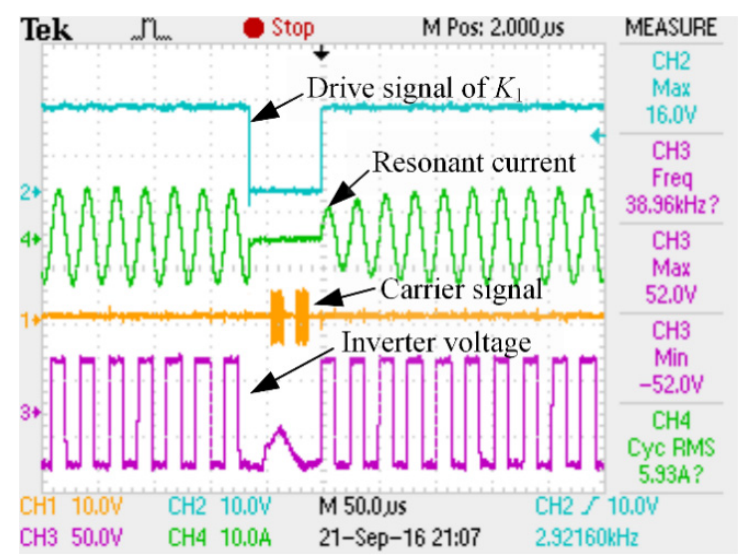

Fig.18. The voltage and current at signal transmission mode 


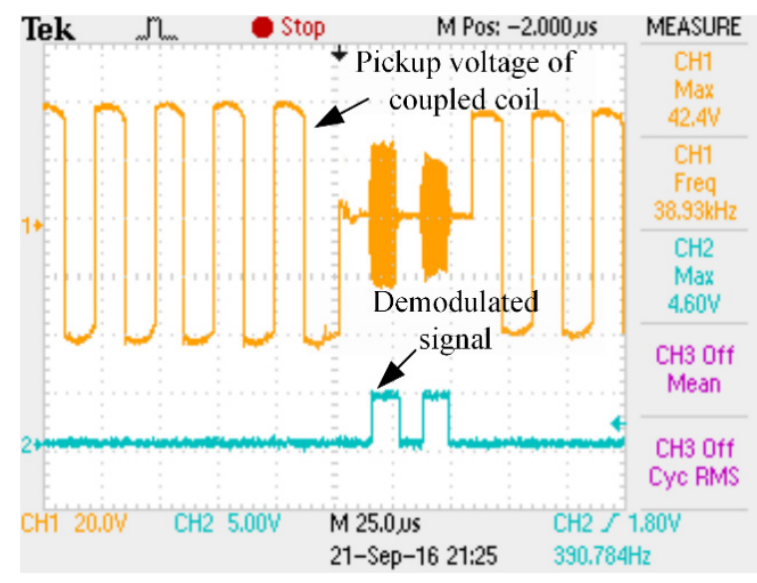

Fig.19. The result of signal demodulation

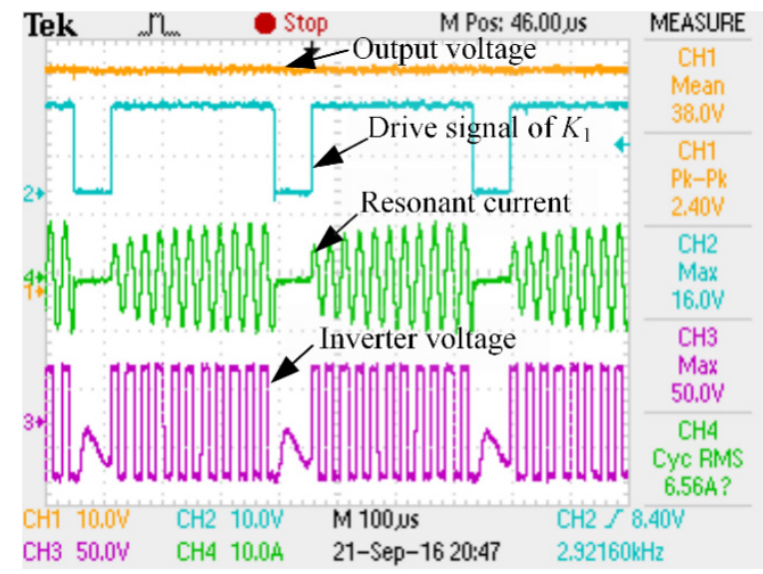

Fig.20. The voltage and current at power transmission mode

The results of signal demodulation and power transmission are given in Fig. 19 and Fig. 20. The signal demodulation is achieved, and the output voltage almost has no fluctuation

As can be seen from the Fig. 20, the time for power transmission is almost $280 \mu \mathrm{s}$, the time for signal transmission is almost $50 \mu \mathrm{s}$, so the baud rate of the setup is:

$$
b p s=\frac{n}{t_{p}+t_{s}}=\frac{4}{(280+50) \times 10^{-6}}=12121 \mathrm{bps}
$$

From the experimental results above, the proposed method of power and signal synchronous transmission based on time-sharing multiplexing of coupled coil is effective.

\section{CONCLUSION}

For many WPT applications, communication between the primary side and the pick-up side is essential. This paper proposed a method to realize the wireless power and signal transmission via the same coupling coil. In the proposed system, the power carrier and signal carrier are transferred via the same coupling coil based on the timesharing multiplexing technique. The operational mode of the system is discussed in detail, and the mathematical model has been presented to analyze the PTE. Experimental results indicate that the baud rate of the setup is 12121. Meanwhile, the relationship between $t_{p}$ and $t_{s}$ is researched to ensure the stability of power transmission and realize the signal transmission. The results obtained from the experimental platform verify the effectiveness of the proposed method.

\section{Acknowledgments}

Thanks for the support by the Science and Technology Project of China Southern Power Grid Corporation under Project GXKJXM20162018 and the project of Research and Development of Power Distribution Intelligent Line Patrol Drone based on RTK High-precision Positioning Wireless Charging Endurance Technology (2018AB16014).

\section{References}

1. S. Raju, R. Wu, M. Chan, and C. P. Yue, "Modeling of Mutual Coupling Between Planar Inductors in Wireless Power Applications," IEEE Trans. Power Electron., vol. 29, no. 1, pp. 473-480, Jan. 2014.

2. D. Ahn and S. Hong. "Wireless power transmission with self-regulated output voltage for biomedical implant," IEEE Trans. Ind. Electron., vol.61. no.5, pp. 2225-2235, May 2014.

3. R. Wu, S. Raju, M. Chan, J. K. O. Sin, and C. P. Yue, "Silicon-embedded receiving coil for highefficiency wireless power transfer to implantable biomedical ICs," IEEE Electron Device Lett., vol. 34, no. 1, pp. 9-11, Jan. 2013.

4. J. Kim, H. C. Son, D. H. Kim, and Y. J. Park, "Optimal design of a wireless power transfer system with multiple self-resonators for an LED TV," IEEE Trans. Con. Electron., vol. 58, no. 3, pp. 775437800, Aug. 2012.

5. S. Y. R. Hui and Wing. W. C. Ho, "A new generation of universal contactless battery charging platform for portable consumer electronic equipment," IEEE Trans. Power Electron., vol.20, no.3, pp 620-627, May 2005.

6. M. Yilmaz and P. T. Krein, "Review of battery charger topologies, charging power levels, and infrastructure for plug-in electric and hybrid vehicles," IEEE Trans. Power Electron., vol. 28, no.5, pp. 2151-2169, May 2013.

7. M. Budhia, J. T. Boys, G. A. Covic, C. Y. Huang, "Development of a single-sided flux magnetic coupler for electric vehicle IPT charging systems," IEEE Trans. Ind. Electron., vol. 60, no. 1, pp. 318328, Jan. 2013.

8. M. McDonough, P. Shamsi, B. Fahimi, “Application of multi-port power electronic interface for contactless transfer of energy in automotive applications," in Vehicle Power and Propulsion Conference, IEEE, 2011, pp. 1-6.

9. Siqi Li, and Chunting Chris Mi, "Wireless Power Transfer for Electric Vehicle Applications," IEEE 
Journal of Emerging and Selected Topics in Power Electronics., vol. pp, no. 99, pp. 1-14, Apr. 2014.

10. J. Shin, S. Shin, Y. Kim, S. Ahn, S. Lee, G. Jung, S. Jeon, and D. Cho, "Design and implementation of shaped magnetic-resonance-based wireless power transfer system for roadway-powered moving electric vehicles," IEEE Trans. Ind. Electron., vol. 61, no.3, pp. 1179-1192, Mar. 2014.

11. G. B. Tao, J. F. Xu, G. J. Fu, and Z.Wang, "Research on contactless power transfer efficiency of rotary steerable drilling tool," in Proc. Int. Conf. Intell. Control Inf. Process., 2011, pp. 536-539.

12. V. J. Brusamarello, Y. B. Blauth, R. de Azambuja, I. Muller, and F. R. de Sousa, "Power transfer with an inductive link and wireless tuning," IEEE Trans. Instrum. Meas., vol. 62, no.5, pp. 924-931, May 2013.

13. Z. Wang, Y. Li, Y. Sun, C. Tang, and X. Lv, "Load detection model of voltage-fed inductive power transfer system," IEEE Trans. Power Electron., vol. 28, no.11, pp. 5233-5243, Nov. 2013.

14. D. J. Thrimawithana, U. K. Madawala and M. Neath, "A synchronization technique for bidirectional IPT systems," IEEE Trans. Ind. Electron., vol. 60, no.1, pp. 301-309, Jan. 2013.

15. S. Ping, A. P. Hu, S. Malpas, and D. Budgett, "A frequency control method for regulating wireless power to implantable devices," IEEE Trans. Biomed. Circuits Syst., vol. 2, no.1, pp. 22-29, Mar. 2008.

16. G. X.Wang, P. J.Wang, and W. T. Liu, "Analysis of dual band power and data telemetry for biomedical implants," IEEE Trans. Biomed. Circuits Syst., vol. 6, no. 3, pp. 208-215, Jun. 2012.

17. G. B. Tao, J. F. Xu, G. J. Fu, and Z.Wang, "Research on contactless power transfer efficiency of rotary steerable drilling tool," in Proc. Int. Conf. Intell. Control Inf. Process., 2011, pp. 536-539.

18. J. F. Zhou, Y. Sun, Y. G. Su, X. Dai, and Y. Zhai, "Synchronous transmission of inductively coupled power and signal," Journal of Chongqing Institute of Technology, vol. 23, no. 4, pp. 12-17, Apr. 2009.

19. S. Yong-ho and J. Byung-jun, "Simultaneous data and power transmission in resonant wireless power system," in Proc. Micro. Conf., 2013, pp. 10031005.

20. Y Sun, P. X. Yan, Z. H. Wang and C. S. Tang, "Research on real-time and synchronization transmission of power and data via a shared channel in inductive coupling power transfer system," Proceedings of the CSEE, Vol. 36, no. 19, pp. 51725178, Oct. 2016.

21. X. Dai, R. J. Du, C. S. Tang, Z. H. Wang, Y. Sun, "A 2FSK-Based high-speed signal transmission method for ICPT system," Journal of Southwest Jiaotong University., vol. 48, no. 5, pp. 892-897, Oct. 2013.

22. H. Hao, G. A. Covic, J. T. Boys, "A parallel topology for inductive power transfer power supplies," IEEE Trans. Power Electron., vol. 29, no.3, pp. 1140-1151, Mar. 2014.

23. W. X. Zhong, S. Y. R. Hui, "Maximum energy efficiency tracking for wireless power transfer systems," IEEE Trans. Power Electron., vol. 30, no.7, pp. 4025-4034, Jul. 2015. 